\title{
METODOLOGÍA DE DETECCIÓN DE FALLAS DE UN MOTOR SÍNCRONO
}

\section{METHODOLOGY FOR FAULT DETECTION OF A SYNCHRONOUS MOTOR}

\author{
MSc. Yesenia Restrepo Chaustre*, MSc. José A. Becerra Vargas.* \\ PhD. Aldo Pardo García.** \\ * Universidad Francisco de Paula Santander. \\ Avenida Gran Colombia, No.12E-96, Colsag. Cúcuta Norte de Santander. \\ E-mail: $\{$ yeseniarestrepo, josearmandobv $\}$ ufps.edu.co \\ ** Universidad de Pamplona. \\ Ciudadela Universitaria. Pamplona. Norte de Santander, Tel. (+577) 568 5303, Ext. 163. \\ E-mail: apardo13@hotmail.com
}

\begin{abstract}
This article is part of the area of diagnostics and troubleshooting in industrial processes, focusing their application to synchronous motors to identify incipient faults affecting the main advantages of using these engines. At present, there are many methods of detection and diagnosis of faults, but this research focuses on fault detection by correlation and their classification using artificial neural networks.
\end{abstract}

Keywords: Synchronous motor, mathematical model, fault detection, neural networks.

Resumen: Este artículo se enmarca dentro del área de diagnóstico y detección de fallas en procesos industriales, enfocando su aplicación hacia los motores síncronos para identificar las fallas incipientes que afectan las principales ventajas de utilización de estos motores. En la actualidad, existen muchos métodos de detección y diagnóstico de fallas, pero esta investigación se enfoca en la detección de fallas por correlación y clasificación de las mismas utilizando redes neuronales artificiales.

Palabras clave: Motor síncrono, modelo matemático, detección de fallas, redes neuronales.

\section{INTRODUCCIÓN}

Los motores eléctricos son máquinas que pueden convertir la energía eléctrica en energía mecánica como movimiento rotatorio, con el propósito de que efectúe trabajo útil. Estos motores se fabrican desde pequeñas potencias hasta potencias grandes y con una amplia variedad de velocidades, que pueden ser fijas, ajustables o variables [1].

Por lo tanto, dada la importancia de los motores síncronos como parte de una red eléctrica, el empleo de modelos matemáticos que permitan la predicción de su desempeño, lo convierte en tema de diversas investigaciones para su caracterización $[2,3]$.
En la actualidad existe en la industria una gran demanda de sistemas que incorporan capacidades de detección y diagnóstico de fallos (SDDF). Debido que estas tareas permiten la detección temprana de las fallas en el proceso, corrigiendo desviaciones en la producción, y principalmente anticipándose a posibles situaciones que pongan en peligro la vida humana y el medio ambiente. Es por estos motivos, por lo que en los últimos años ha sido evidente el desarrollo a nivel de investigación y de transferencia de tecnología del campo de la detección y diagnóstico de fallos tanto a nivel nacional como internacional $[4,5,6]$. 
En los últimos treinta años el diagnóstico automático de procesos es un campo de investigación y desarrollo que se puede abordar desde múltiples perspectivas (Ingeniería de Control e Inteligencia Artificial), y con una variedad de técnicas (sistemas expertos, redes neuronales, redes de Petri, razonamiento basado en casos, análisis de señales, observadores, ecuaciones de redundancia analítica, entre otros) en búsqueda de que al implementar los Sistemas de Detección y Diagnóstico de Fallas, se pueda disminuir el riesgo y además aumentar la Confiabilidad, Disponibilidad, reducir costos de mantenimiento, y aumentar la vida útil de los sistemas, en especial interés la vida útil del motor síncrono, motivo de la presente investigación.

\section{MARCO TEÓRICO}

\subsection{Máquina síncrona}

Las máquinas síncronas son máquinas eléctricas cuya velocidad de rotación $\mathrm{n}$ (rpm) está vinculada rígidamente con la frecuencia $f$ de la red de corriente alterna con la cual trabaja, de acuerdo con la expresión:

$$
n=\frac{60 f}{P}
$$

Donde $P$ es el número de pares de polos de la máquina [7]. Las máquinas síncronas, como cualquier otro convertidor electromecánico de la energía, están sometidas al principio de reciprocidad electromagnética, pudiendo funcionar tanto en régimen generador como en régimen motor. Estas máquinas pueden cumplir tres diferentes funciones: Generadores o alternadores, Motores, Condensador síncrono.

\subsection{Circuito equivalente del generador síncrono}

Considerando $\mathrm{E}_{\mathrm{A}}$ el voltaje interno generado en una fase del generador síncrono, no es normalmente el voltaje que se presenta en las terminales del generador $V_{\theta}$ voltaje de salida. Hay factores que ocasionan la diferencia que hay entre $\mathrm{E}_{\mathrm{A}} \mathrm{y} V_{\theta}$.

- La distorsión del campo magnético del entrehierro debida a la corriente que fluye en el estator, llamada reacción de armadura.

- La autoinducción de las bobinas de armadura.

- La impedancia síncrona.

- El efecto de la forma del rotor de polos salientes.

\subsection{Sistemas de detección y diagnóstico de fallas}

La posibilidad de fallas incipientes es inherente en las máquinas eléctricas debido al desgaste involucrado en la conversión de energía eléctrica a mecánica y viceversa [9]. Monitoreando este desgaste de manera oportuna, pueden mejorarse los costos de mantenimiento y la confiabilidad de los sistemas. Las redes neuronales pueden ser entrenadas por el conocimiento de un sistema experto para mejorar la detección de la falla y después de ser entrenada, la red deberá contener el conocimiento necesario para la detección de las fallas sin ayuda del sistema experto [10].

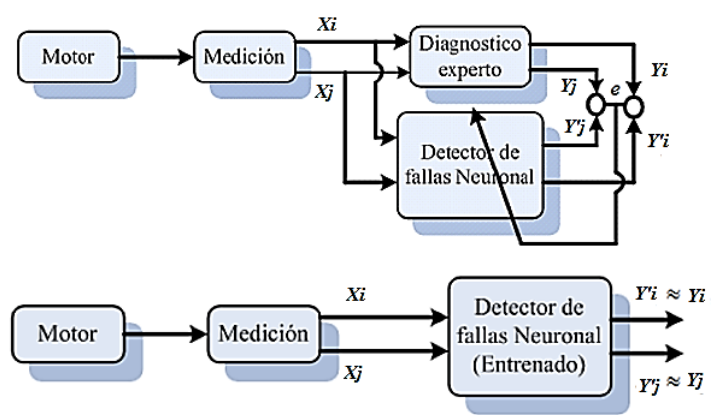

Fig. 1. Proceso de detección y diagnóstico de fallas en el motor (Fuente: [10]).

\section{DESARROLLO METODOLÓGICO}

\subsection{Pruebas y obtención de parámetros}

Las pruebas realizadas a la máquina síncrona proporcionan características eléctricas y mecánicas de ésta, son parámetros que al tenerlos como referencia se pueden utilizar para comparaciones en pruebas posteriores. Así, se puede determinar qué factores afectan en el funcionamiento $y$ características de la máquina, para poder tomar decisiones sobre la detección de fallas y prevención de futuros daños. Entre las pruebas realizadas tenemos: prueba de vacío, de corto circuito y de pérdidas mecánicas.

El circuito equivalente por fase resultante del motor síncrono trifásico DL30190 se muestra en la figura 2, la inductancia del circuito de campo es despreciable comparada con $R_{F}\left(L_{F}=0\right)$. 


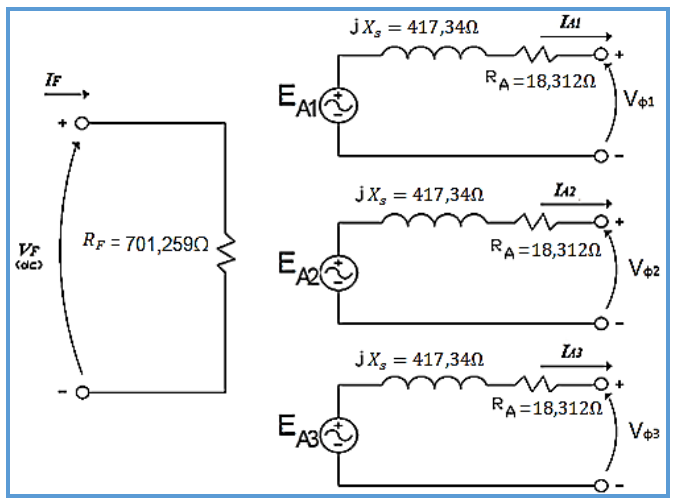

Fig. 2. Circuito equivalente del motor síncrono trifásico DL30190.

\subsection{Modelo de la máquina síncrona de dos ejes y representación en diagrama de bloques.}

Utilizando la transformación de Park [11, 12, 13] y utilizando las coordenadas rotóricas, conocidas como eje $d$ y $q$ (eje directo y eje de cuadratura) [11, $12,13,14]$, se obtienen las reactancias directa y de cuadratura, donde estas reactancias se definen iguales en un motor síncrono de rotor liso, y por tal característica se obtienen de la reactancia sincrónica.

Por lo tanto al conocer la reactancia sincrónica, Xs, tenemos las reactancias directa y de cuadratura, $X \mathrm{~d}$ y Xq [9, 12]: $X_{s}=\left(X_{d}+X_{q}\right) / 2$

Luego, utilizando los datos obtenidos en la prueba de pérdidas mecánicas, en el hierro y pérdidas adicionales del ítem 3.1, se procede a hallar la reactancia de magnetización del núcleo $\left(X_{m}\right)$ y resistencia del núcleo $\left(R_{F e}\right)$.

$R_{F e}=20.82 \Omega, X_{m}=337.82 \Omega$

Tomando los valores de la curva de magnetización: $E_{f}=V_{f}=381 \mathrm{~V}$

Desarrollando la siguiente ecuación, el vector de derivadas de las variables de estado $p[\mathrm{i}]$ :

$$
p[i]=[L]^{-1}\{[V]-[|R|+w|G||i|]\}
$$

Se obtiene la siguiente representación de la máquina sincrónica en variables de estado:

$$
p\left[\begin{array}{c}
i_{d} \\
i_{q} \\
i_{f}
\end{array}\right]=\left[\begin{array}{ccc}
-\frac{R_{e}}{L_{d}^{\prime}} & \frac{w L_{q}}{L_{d}^{\prime}} & -\frac{R_{f}}{L_{d f}} \\
-\frac{w L_{d}}{L_{q}} & -\frac{R_{e}}{L_{q}} & -\frac{w L_{d f}}{L_{q}} \\
-\frac{R_{e}}{L_{d f}^{\prime}} & \frac{w L_{q}}{L_{d f}} & -\frac{R_{f}}{L_{f}^{\prime}}
\end{array}\right]\left[\begin{array}{l}
i_{d} \\
i_{q} \\
i_{f}
\end{array}\right]+\left[\begin{array}{ccc}
\frac{1}{L_{d}^{\prime}} & 0 & \frac{1}{L_{d f}^{\prime}} \\
0 & \frac{1}{L_{q}} & 0 \\
\frac{1}{L_{d f}^{\prime}} & 0 & \frac{1}{L_{f}}
\end{array}\right]\left[\begin{array}{c}
V_{d} \\
V_{q} \\
V_{f}
\end{array}\right](6)
$$

De esta representación matricial se pueden obtener las siguientes funciones de transferencia de primer orden:

$$
\begin{aligned}
& i_{d}=\frac{1 / \mathrm{R}_{\mathrm{e}}}{\left(L_{d}^{\prime} / \mathrm{R}_{\mathrm{e}}\right) p+1}\left[w L_{q} i_{q}+R_{f} \frac{L_{d f}}{L_{f}} i_{f}+v_{d}-\frac{L_{d f}}{L_{f}} v_{f}\right] \\
& i_{d}=\frac{1 / \mathrm{R}_{\mathrm{e}}}{\left(L_{q} / \mathrm{R}_{\mathrm{e}}\right) p+1}\left[-w L_{d} i_{d}+w L_{d f} i_{f}+v_{q}\right] \\
& i_{f}=\frac{1 / \mathrm{R}_{f}}{\left(L_{f} / \mathrm{R}_{f}\right) p+1}\left[-R_{e} \frac{L_{d f}}{L_{d f}^{\prime}} i_{d}+w \frac{L_{q} L_{f}}{L_{d f}^{\prime}} i_{q}+\frac{L_{f}}{L_{d f}^{\prime}} v_{d}+v_{f}\right]
\end{aligned}
$$

Donde las tensiones son las variables de control y las corrientes las variables de estado. En Simulink ${ }^{\circledR}$ del entorno Matlab $^{\circledR}$, se representa este modelo en diagrama de bloques, como se muestra en la fig. 3 .

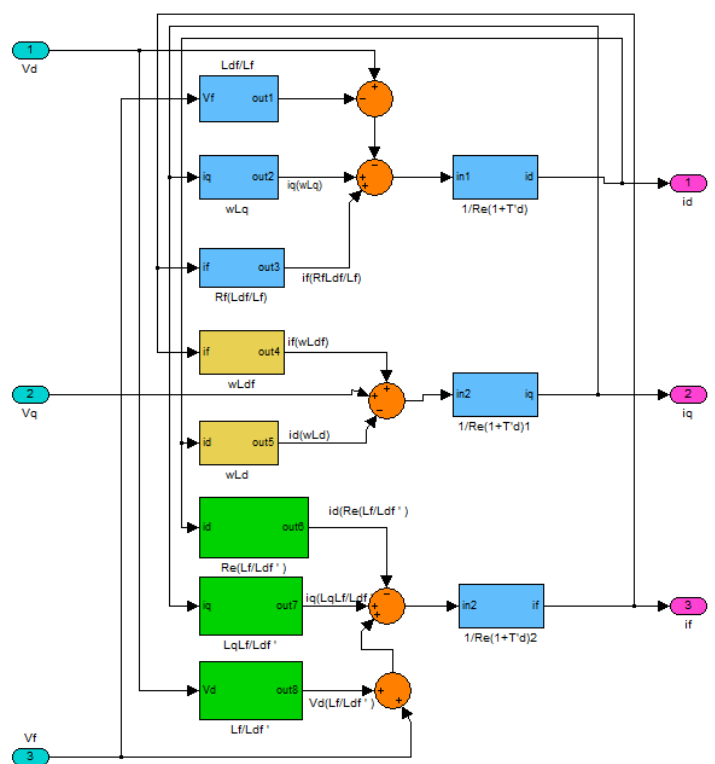

Fig. 3. Representación en bloques de Simulink ${ }^{\circledR}$ de la máquina síncrona de rotor liso, sin devanados amortiguadores.

En la figura 4, se muestran las señales de entrada al subsistema de la máquina síncrona, representadas por dos ondas sinusoidales y una señal constante, obtenida de Library: Simulink/Sources para visualizar las variables de salida con el scope de Simulink.

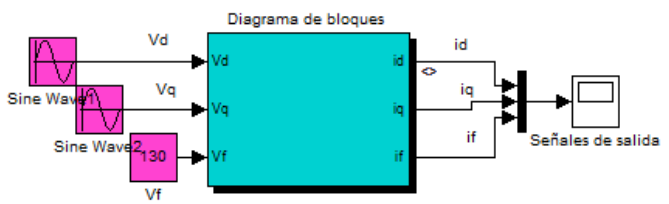

Fig. 4. Representación del subsistema con sus respectivas señales de entrada. 
En la figura 5, se observan las variables de salida del modelo, representada en sus respectivas corrientes de cuadratura y de eje directo.

\subsection{Metodología de diagnóstico de fallas utilizando redes neuronales}

Para la implementación del sistema de diagnóstico de fallas en necesario realizar la identificación propia del sistema.

Dependiendo de la técnica o proceso con el cual se identifique el modelo, entre los cuales tenemos:

- Modelado de caja negra (BlackBox modelling), basada exclusivamente en los datos medidos.

- Modelado de caja blanca (WhiteBox modelling), basada puramente en el modelado físico del sistema.

- Modelado de caja gris (GrayBox modelling), cuando existe un cierto nivel de conocimiento del sistema y es utilizado para mejorar el modelo empírico $[10,15]$.

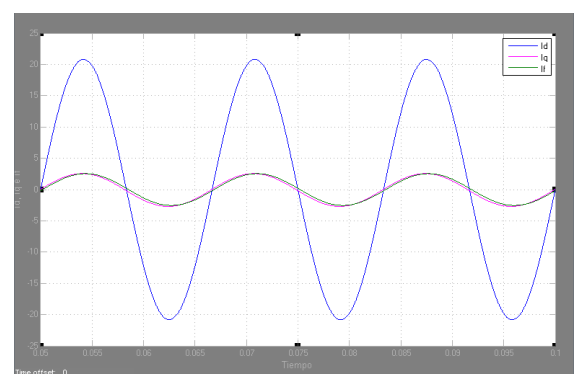

Fig. 5. Señales de respuesta del sistema, variables de salida $i_{d}, i_{q}$ e $i_{f}$ del modelo.

Para la presente investigación se utiliza el WhiteBox modelling, basado en el modelo físico caracterizado del sistema, en este caso el motor síncrono DL30190 ubicado en el Laboratorio Especializado de Máquinas Eléctricas de la Universidad Francisco de Paula Santander, LE102. Los requerimientos para una Red Neuronal (RN) son menores en comparación con los procedimientos clásicos, ya que el usuario debe especificar la característica de la estructura de la relación entre las entradas y las salidas, mientras que para la identificación con las RN sólo se debe especificar la topología de la red [16]. La RN no intenta asumir ninguna estructura de la relación entrada-salida, ella la crea a partir de los ejemplos proporcionados durante el entrenamiento. Lo que se pide y necesita de la $\mathrm{RN}$ en este caso, es que internamente consiga un modelo capaz de reproducir unos valores numéricos que se asemejen a los valores de algún sistema físico [16], en éste

caso al sistema físico presentado con posibles fallas.

\subsubsection{Identificación con red neuronal Multilayer Perceptron}

La red neuronal utilizada es una red neuronal Multilayer-Perceptron (MLP), entrenada por el algoritmo BackPropagation, utilizadas en problemas de predicción, clasificación, reconocimiento de patrones, estimación de parámetros y resolución de señales.

Para la identificación de sistemas dinámicos la red neuronal utiliza un lazo de realimentación (feedback) con las salidas anteriores del sistema o planta, considerándolas como otras entradas adicionales a la red, a lo que se le conoce como entrenamiento supervisado. Para este proceso de identificación de los parámetros de la planta o modelo matemático del motor se utilizará una red neuronal de tres capas (entrada, oculta y salida) y un bloque adicional de retardos que se añaden como entradas provenientes de la salida del sistema o modelo matemático del motor.

\subsubsection{Identificación del modelo con redes neuronales utilizando Matlab ${ }^{\circledR}$ Simulink}

Para la identificación del sistema o planta con redes neuronales se debe disponer de un conjunto de datos, adquiridos de forma experimental o mediante algún modelo de Simulink [16]. En el presente trabajo se utiliza el modelo implementado en Simulink.

Adicionalmente se usa el bloque "To Workspace" para generar el arreglo de datos necesarios para el entrenamiento de la red neuronal. Igualmente, se creó un archivo de extensión (.mat), que contiene éstos datos, los cuales se utilizan, tanto para el entrenamiento de la red como para la validación de la misma, ver figura 6 . Una vez generados los datos se procede a crear y entrenar la Red Neuronal, para ello se utiliza el siguiente código extensión .asm:

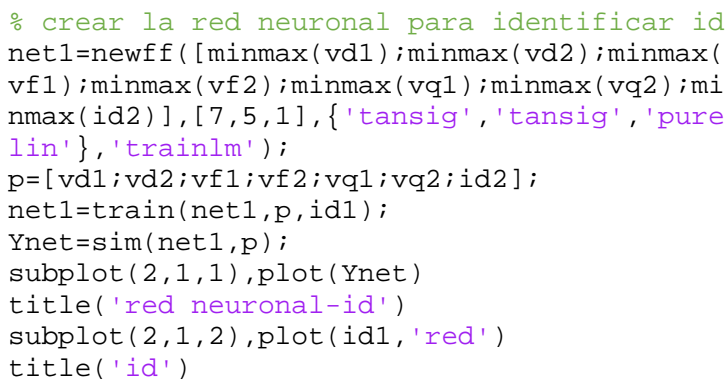




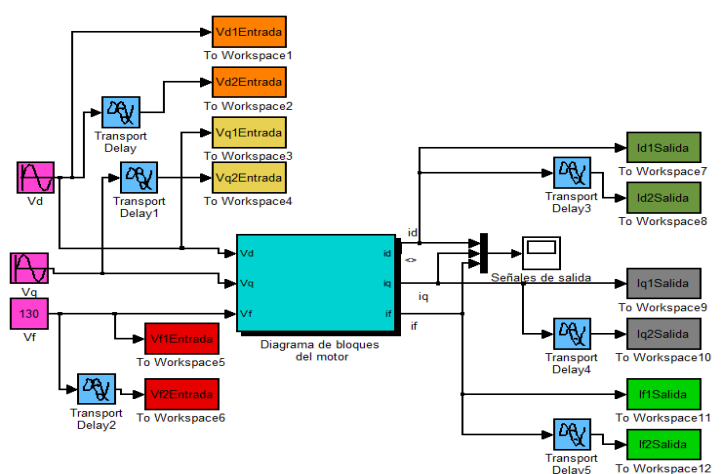

Fig. 6. Esquema en Simulink para generar datos

Para la creación de las neuronas de la capa de entrada y la oculta se utilizó la función de activación o de transferencia "Tansig", ya que si para ello se utilizaba la función "Losing" o la función "Purelin", presentaban unos gradientes que alejaban a la red de su aproximación ideal, mientras, que para la capa de salida se utilizó la función de activación "Purelin".

En el entrenamiento de las redes neuronales fue necesario utilizar la función "LevenbergMarquardt", ya que éste método de entrenamiento presenta mejor rendimiento que los otros, inclusive que el "Gradiente Descendente", sin embargo, si requiere más memoria que los otros métodos [16].

En la figura 7, se observa la comparación que se realizó del entrenamiento de la red neuronal con respecto a la data original del modelo, teniendo como resultado que la señal presenta un comportamiento similar a la identificada con la Red Neuronal Feedforward (Feedforward Neural Network), por consiguiente el entrenamiento de la red es satisfactorio.

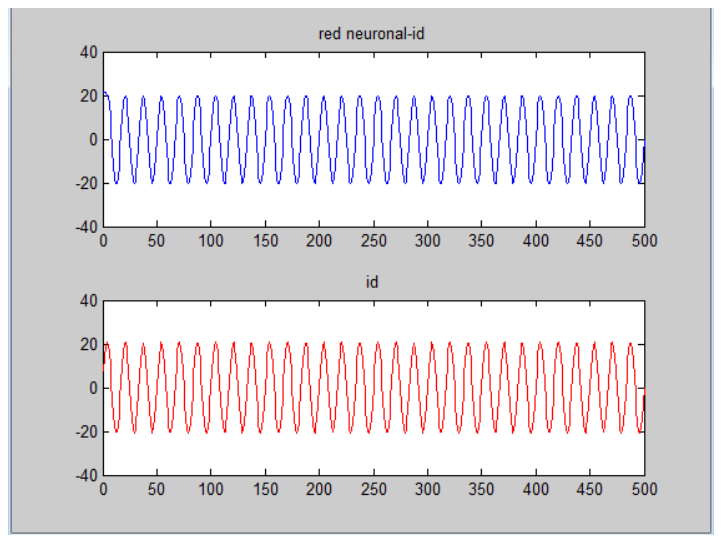

Fig. 7. Gráfica de la corriente del eje directo, simulada por la red neuronal y la del modelo matemático del motor.

\subsubsection{Identificación y clasificación de fallas}

Las fallas en las máquinas eléctricas, en la mayoría de los casos no se manifiestan de manera intempestiva sino gradualmente, esto hace posible la detección de muchas fallas antes de que sus consecuencias resulten catastróficas. En éstas últimas décadas se han desarrollado nuevas técnicas de detección de fallas que permiten obtener diagnósticos precisos, entre éstas tenemos: el análisis de vibración, análisis espectral de corrientes (MCSA), análisis de dispersión del flujo axial (AF) y las más recientes que combinan modelos de simulación del comportamiento de las fallas y la aplicación de redes neuronales para identificar fallas; ésta última es la técnica en la que se centra este artículo.

En el presente trabajo se modelan las fallas por corto circuito brusco y por saturación de la máquina síncrona. Para la identificación del sistema o planta con redes neuronales, cuando ocurre una falla de corto circuito se procede como en el inciso anterior; se implementa el modelo en Simulink, como se muestra en la figura 7 , en el que se representa la falla o corto circuito de la red con una señal escalón simulando ésta el cambio brusco de tensión. De igual manera se utiliza el boque "To Workspace" para generar el arreglo de datos necesarios para el entrenamiento de la red neuronal. Este arreglo de datos se guarda en un archivo de extensión (.mat), para utilizarlos en el entrenamiento y la validación de la red.

Una vez generados los datos se procede a crear y entrenar la Red Neuronal, según el programa:

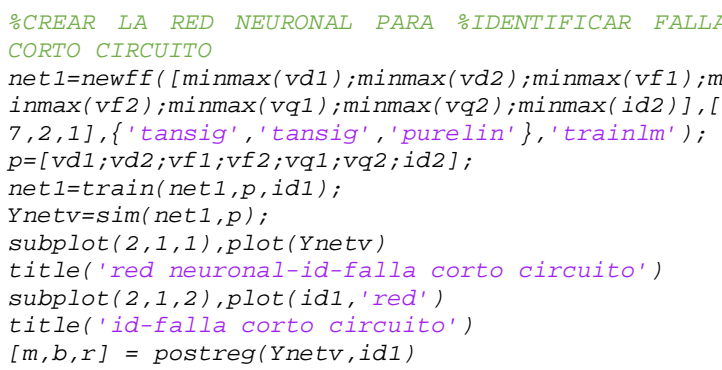

Se utilizaron 7 neuronas de entrada, 2 neuronas ocultas y una de salida; porque al utilizar la misma cantidad de neuronas creadas en el programa anterior no identificaba bien el sistema con la falla de corto circuito. Para el entrenamiento de las redes neuronales se utilizó la función "LevenbergMarquardt". En la siguiente figura se ilustra la simulación de la red entrenada. 
De la identificación de la red, con falla de corto circuito, se tiene una correlación de los datos obtenidos del modelo del motor con los datos obtenidos con la red de 0.9985 (ver figura 9), como se muestra a continuación:

$$
m=0.9971 ; b=-7.1333 e-006 ; r=0.9985
$$

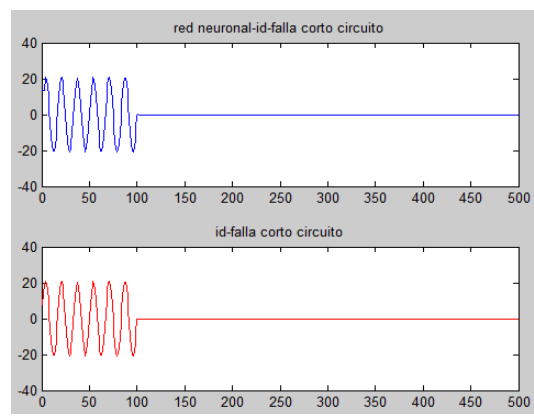

Fig. 8. Gráfica de la corriente del eje directo, simulada por la red neuronal y la del modelo matemático del motor cuando presenta una falla de corto circuito.

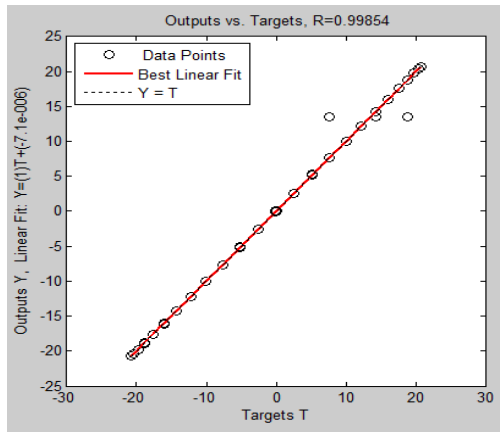

Fig. 9. Gráfica de correlación de los datos simulados falla corto circuito.

\subsubsection{Detección de fallas incipientes en el motor}

La estrategia más común es elegir el modelo que provea la mejor predicción un paso adelante en términos de un error cuadrático mínimo entre las salidas del sistema y las estimadas del modelo propuesto. Este proceso es llamado entrenamiento o aprendizaje. La validación radica en usar el modelo estimado/entrenado e investigar si cumple con los requerimientos necesarios $[17,10]$. Inicialmente se definen la cantidad de fallas a identificar, las entradas y salidas del sistema, éstos parámetros ya se obtuvieron en los incisos anteriores del presente artículo; las fallas que se van a identificar en esta propuesta son las fallas por corto circuito brusco y saturación del motor síncrono; las entradas y salidas ya se simularon en el modelo matemático del motor síncrono el cual fue caracterizado e identificado con una red neuronal. Por lo tanto, del diagrama de bloques ya están definidos los datos e identificado el modelo de las fallas. Seguidamente, se definen las reglas en la toma de decisiones para el diagnóstico de fallas y la validación del sistema [10, 15, 18].

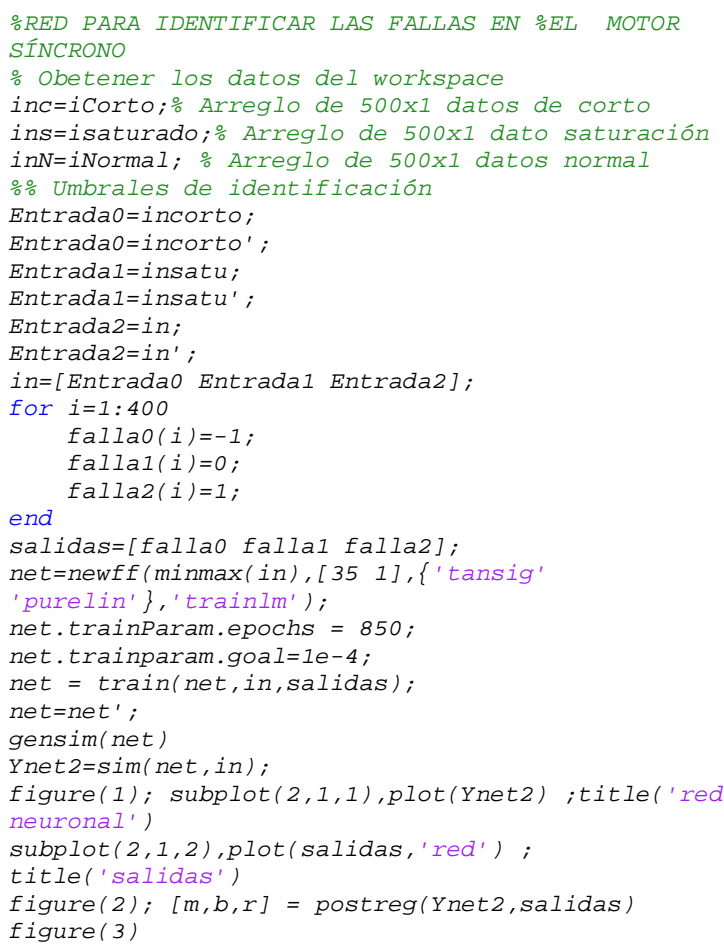

Para la creación de las neuronas de la capa de entrada y la salida se utilizó la función de activación o de transferencia "Tansig" y "Purelin", y se utilizaron 35 neuronas de entrada, y una de salida. Para el entrenamiento de las redes neuronales se utilizó la función "LevenbergMarquardt".

En la siguiente figura se ilustra la simulación de la red entrenada y la gráfica de la data de entrenamiento.

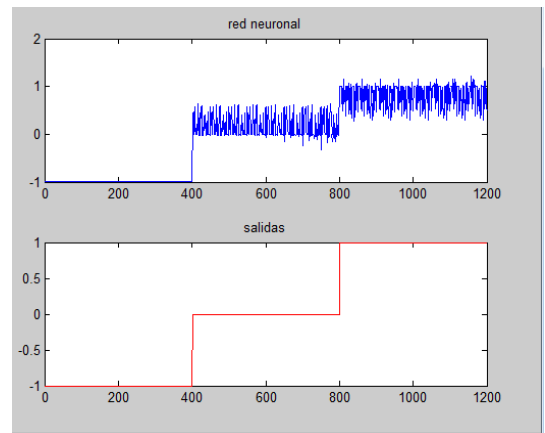

Fig. 10. Gráfica de la clasificación e identificación de fallas, simulada por la red neuronal y las salidas esperadas del sistema. 


\section{VALIDACIÓN}

Para la comparación y validación se utiliza como base el modelo de:

- Kwok-Wai L.e, del proyecto titulado: "Phasedomain synchronous generator model for transients simulation" [19].

- El modelo de Andrés, S.H y Carlos, G.R, del proyecto titulado: "Validación del modelo DQO de la máquina síncrona para estudios de transitorios electromagnéticos" [12].

A continuación se muestra la corriente de fase sin saturación del modelo de Kwow-Wai L. [19] y la obtenida por el modelo de Andrés, S. H. [12].

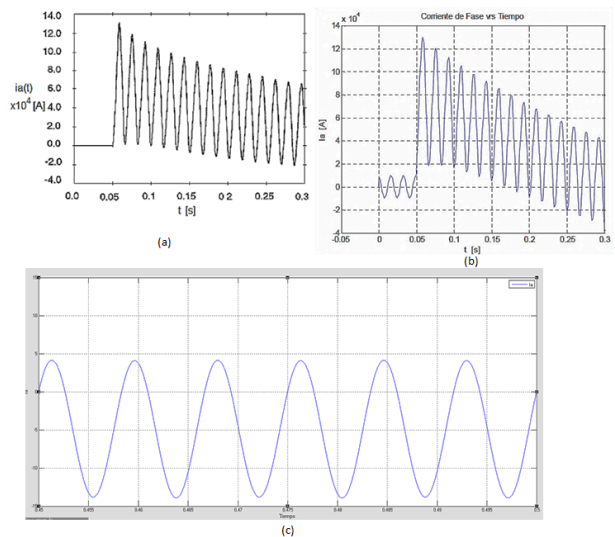

Fig. 11. Corriente de la fase a, o coordenada primitiva: (a) Modelo Kwok - Wai Louie [19];

(b) Modelo de Andrés, S.H. [12]; (c) Modelo DL30190.

En la figura 11, se presenta la comparación de las curvas de la corriente de la fase a del modelo desarrollado en este libro para el motor DL30190, con el presentado por Kwow-Wai Louie [19] y el modelo presentado por Andrés S. H. [12], todas las anteriores presentan un comportamiento asimétrico lo que las hace muy parecidas. Más sin embargo, las corrientes del inciso a) y b) presentan oscilaciones diferentes a la presentada por el modelo desarrollado, y ésta diferencia se debe a que los modelos [19] y [12] son presentados con una perturbación inicial hasta que ésta señal se estabiliza.

\subsection{Validación de la red neuronal de identificación del modelo del motor síncrono}

Para la validación preparamos los datos que vamos a utilizar, para ello de manera aleatoria dividimos el conjunto de datos en cuatro grupos, de los cuales un grupo se utiliza para crear la red, el otro para entrenar la red y el tercero para validar la red.
De la validación de los datos anteriores se obtiene: $m=1.0000 ; \quad b=3.9792 e-007 ; \quad r=1.0000 ;$

Teniendo en cuenta el resultado de la regresión $(\mathrm{r}=1)$, se observa una perfecta correlación entre la red neuronal y los datos de validación, por lo tanto, la red neuronal que se utilizó para identificar al modelo matemático cumple perfectamente con las características del mismo.
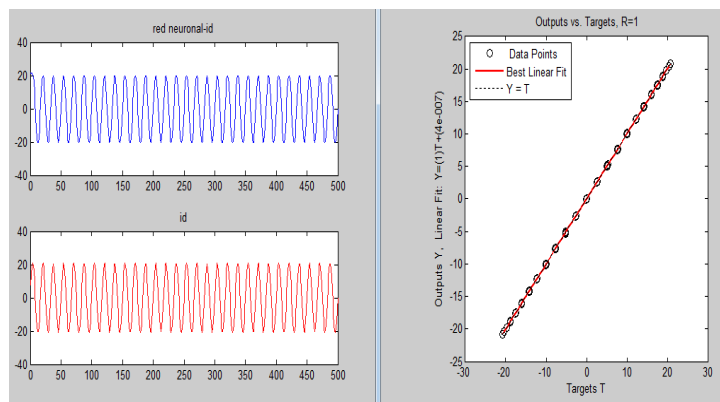

Fig. 12. Validación de la red neuronal que identifica el modelo matemático.

\subsection{Validación de la red neuronal de} identificación y clasificación de fallas del motor

Para la validación preparamos los datos que vamos a utilizar, para ello de manera aleatoria dividimos el conjunto de datos en cuatro grupos, de los cuales un grupo se utiliza para crear la red, el otro para entrenar la red y el tercero para validar la red.

De la validación de los datos anteriores se obtiene: $m=0.9992 ; \quad b=-1.6269 e-005 ; \quad r=0.9996 ;(12)$

El resultado de la regresión $(\mathrm{r}=0.9996)$, da muestra de una buena correlación entre la red neuronal y los datos de validación, ya que es aproximadamente igual a 1, por lo tanto, la red neuronal que se utilizó para identificar y clasificar la falla por corto circuito al motor síncrono cumple adecuadamente con las características del mismo.

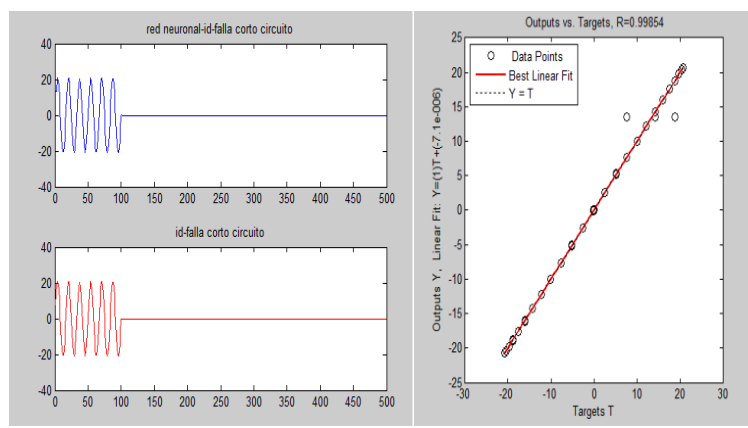

Fig. 13. Validación de la red neuronal que identifica la falla por saturación del motor. 
4.3 Validación de la red neuronal de identificación y clasificación de fallas del motor

Para validar el modelo de clasificación de fallas se utilizó una data que facilita el autor [11], en ésta data el autor (Aller) entrega el código fuente con el cual se generan los datos debido a una falla por corto circuito. En la figura 14 se muestra la validación de falla por corto circuito, en el que se observa una salida marcada en un nivel de uno, ya que esta falla se clasificó en la red neuronal con un valor de "1" cuando éste ocurriera. Para la validación por saturación se utilizaron los datos extraídos del modelo del presente proyecto, como se muestra en la figura 15.

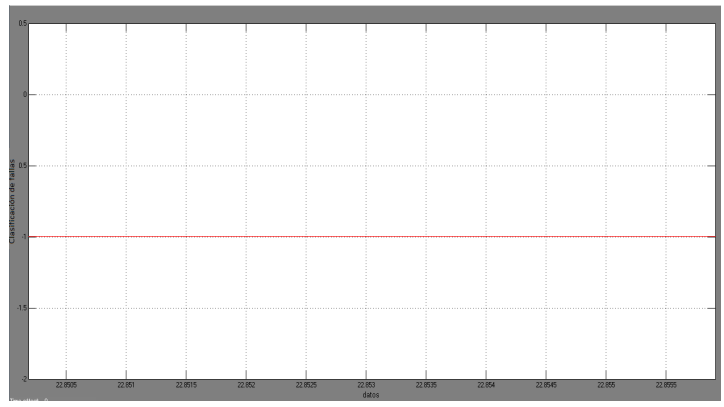

Fig. 14. Validación de la red neuronal que identifica la falla por corto circuito.

La salida oscila alrededor del valor "0", valor con el que se caracterizó o clasificó la falla por saturación en la red neuronal.

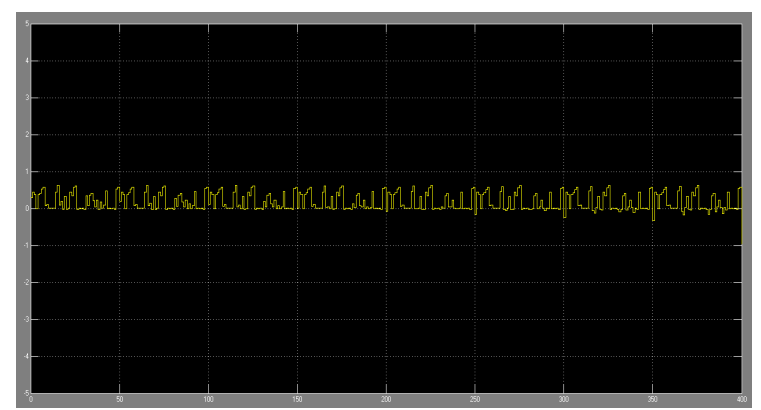

Fig. 15. Validación de la red neuronal que identifica la falla por saturación.

\section{CONCLUSIONES}

Utilizando las pruebas experimentales se caracterizó el motor síncrono DL30190, ubicado en los Laboratorios Especializados de la Universidad Francisco de Paula Santander. Parámetros necesarios para modelar matemáticamente el mismo.
Las redes neuronales perceptron multicapa, con al menos una capa oculta y una función de activación puede aproximar una gran diversidad de funciones no lineales. Los parámetros de la red neuronal se ajustan para modelar el comportamiento de una función dada.

En este proyecto la red neuronal que se ajustó al modelo que simula el motor síncrono es la red "newff", con función de activación "Tasing", "purelin" y función de entranamiento "LevenberMarquar"; éste método de entrenamiento de redes neuronales requiere más memoria que los otros métodos, pero presenta una respuesta más exacta como se muestra en los anexos.

Para la clasificación de las fallas se utiliza una red "newff", pero con una mayor cantidad de neuronas de entrenamiento para la capa de entrada para clasificar e identificar adecuadamente las fallas. En ambos casos de redes neuronales, identificación del modelo y clasificación de las fallas presentaba una correlación cercana a uno.

\section{REFERENCIAS}

[1]. Luna Moreno, H. "Pruebas y obtención de parámetros de la máquina síncrona". Universidad Michoacana de San Nicolás de Hidalgo. México. 2008.

[2]. Aller, J. M. Conversión de energía eléctrica. Universidad Simón Bolívar. 2009.

[3]. M. Cisneros. M. A. Arjona. F.S Sellschopp. Identificación paramétrica de un generador síncrono a partir de la prueba de escalón de voltaje. Científica, Vol. 14, No. 1. pp. 33-39. Enero - Marzo, 2010.

[4]. Hernandez Cely, M. M. "Estudio para detección y diagnóstico de fallas en el proceso del generador de vapor de la central termoeléctrica trmotasajero colgener”. 2010.

[5]. Rubio Ávila, J. Modelado y control para una clase de sistemas no lineales desconocidos en tiempo discreto. 2007

[6]. Gertler, J. J. Fault Detection and Diagnosis in Engineering Systems. New York: Marcel Dekker. 1998.

[7]. Fraile Mora, J. Máquinas Eléctricas. Quinta Edición. Mc Graw Hill. 2003.

[8]. Chapman, S. J. Máquinas Eléctricas. Cuarta Edición. Mc Graw Hill. 2005

[9]. Chow, M. Y.; Sharpe, R. N. and Hung, J. C. On the Application and Design of Artificial Neural Networks for Motor Fault Detection- 
Part I. IEEE Transactions on Industrial Electronics, Vol. 40. No. 2. April. 1993.

[10]. Cuevas Silva, O. Diagnóstico de fallas en un sistema no lineal empleando redes neuronales, México, 2009.

[11]. Aller, J. M. Universidad Simón Bolívar. Máquinas eléctricas rotativas: Introducción a la teoría general. Editorial Equinoccio, 2008.

[12]. Santos H., A. M. y Gómez R., C. A. Validación del modelo DQ0 de la máquina síncrona para estudios de transitorios electromagnéticos, 2010.

[13]. Pérez Londoño, A. M. y Romero R., Y. A. Medición indirecta de algunos parámetros de la máquina síncrona a partir de la medida del ángulo par, 2007.

[14]. Ohm, D. Y. Dynamic model of PM synchronous motors. Drivetech, Inc, Blacksburg, Virginia.
[15]. Korbics J., P. K. Artificial Neural Network to fault diagnosis. Wydawnictwa NaucowoTechniezne WNT, Polonia, 2002.

[16]. Arafet P., P.; Chang M., F.; Torres A., M.; Dominguez A., H. Métodos de identificación dinámica, Universidad de Oriente, Junio, 2008.

[17]. Norgaard, M. Neural Networks for modelling and control of Dynamic Systems. Springer, Glasgow, Scotland, U.K., 2000.

[18]. Ruz-Hernández; J. A., Sánchez, E. N.; Suárez, D. A. Neural Network-based Scheme for Fault Diagnosis in Fossil Electric Power Plants, Proceedings of International Joint Conference on Neural Networks, Montreal, Canada, July 31 - August 4, 2005.

[19]. Kwok-Wai, L. Phase-domain synchronous generator model for transients simulation, April, 1995. 